\title{
Frequency and Parameter Estimation of Multi-Sinusoidal Signal
}

\author{
P. Petrović \\ Technical Faculty Čačak, Department for Electronics, University of Kragujevac, Svetog Save 65, 32000, Čačak, Serbia, \\ predragp@tfc.kg.ac.rs
}

\begin{abstract}
Estimating the fundamental frequency and harmonic parameters is basic for signal modeling in a power supply system. This paper presents a complexity-reduced algorithm for signal reconstruction in the time domain from irregularly spaced sampling values. Differing from the existing parameter estimation algorithms, either in power quality monitoring or in harmonic compensation, the proposed algorithm enables a simultaneous estimation of the fundamental frequency, the amplitudes and phases of harmonic waves. The reduction in complexity is achieved owing to completely new analytical and summarized expressions that enable a quick estimation at a low numerical error. It is proved that the estimation performance of the proposed algorithm can attain Cramer-Rao lower bound (CRLB) for sufficiently high signal-to-noise ratios. The proposed algorithm can be applied in signal reconstruction, spectral estimation, system identification, as well as in other important signal processing problems. The simulation and experimental results verify the effectiveness of the proposed algorithm.
\end{abstract}

Key words: Band-limited signals, fundamental frequency and Fourier coefficient estimation, analytical solutions, signal reconstruction, time domain

\section{INTRODUCTION}

$\mathbf{L}$ ITERATURE ON ELECTRICAL parameter measurement techniques for power system applications is both voluminous and generally accessible. The magnitude estimation of a power system signal has been an important area of research for the past few decades, and the methods have almost been standardized for the signals with known frequencies. The electrical parameter measurement of a fixed-frequency signal is a straightforward task. However, if the frequency is not known a priori, it becomes a very difficult task to accurately measure the amplitude and phase. Various numerical algorithms for power measurements are sensitive to frequency variations.

In an electric power system, an increase or decrease in frequency occurs due to disturbances in the power system. Large blocks of load are connected or disconnected, or large sources of generation go offline. Frequency variations are much more likely to occur for the loads that are supplied by a generator isolated from the utility systems (islands). Any frequency deviation from the nominal value of 50 or $60 \mathrm{~Hz}$ can substantially degrade the performance of the measurement devices that operate based on assumption of constant frequency.

Spectrum estimation of discretely sampled processes is usually based on procedure employing the Fast Fourier Transform (FFT). The FFT is a computationally efficient algorithm for computing the Discrete Fourier Transform (DFT). However, although the FFT is quite efficient under fixed-frequency conditions, it does not offer very good performance unless the sampling frequency and the fundamental frequency of the signal are synchronized. It is well known that FFT loses its accuracy under desynchronization and nonstationary conditions, whereas the fundamental/harmonic frequency may vary over time. These errors appear due to the orthogonal finite-impulseresponse (FIR) filters having different magnitude gains at frequencies other that the nominal power frequency [1] and because the frequencies of harmonics are equal to zero of the frequency response of the FIR filter with rectangular window, which is used in the DFT algorithms. These performance limitations are particularly troublesome when analyzing short data records, which frequently occur in practice because many measured processes are brief.

To better satisfy the periodicity requirement of the FFT process, time-weighting functions, called windows and/or correction interpolation algorithms, are used [2, 3]. In this way, however, the error can only be reduced but not removed. If a window is not used, then the synchronization to the grid fundamental frequency is mandatory. Unfortunately, phase-locked loop (PLL), as a traditional synchronization method, has a rather long response time, particularly in the presence of the transient phenomena on the input signal, such as the power supply frequency variations or the phase jumps.

In addition to the disadvantages related to the synchronization of the sampling frequency with the frequency of the signal, the FFT has disadvantages caused by frame implementation. Thus, the FFT processes entire frames of data and cannot provide in-between data. If the calculation is done in a sliding mode, i.e. the FFT is repeatedly applied to a frame of $N$ elements computing of the last $N$-1-shifted elements of the previous frame and a single new element, then FFT requires intensive computational effort, which complicates its integration in low-cost microcontrollers.

A Newton-type recursive numerical algorithm that also considers the system frequency as an unknown signal model parameter to be estimated has been proposed in [4]. It simultaneously estimates the frequency and spectra of the power system. This approach solves the problem of sensitivity to frequency variations. By the introduction of power frequency in the vector of unknown model parameters, the signal model becomes nonlinear, so strategies of nonlinear estimation are used. The recursive algorithm form is improved with a strategy of sequential tuning of the forgetting factor. By this, the proposed 
algorithm convergence and accuracy are significantly improved.

If the generator and the acquisition device are not synchronized, then the FIR filter with optimized frequency responses, which do not need synchronization, can be designed by the least-square (LS) technique [5]. In this case, the computational load is higher than in the synchronized case. The LS design method for large-order filters requires a considerable amount of computation that may not be completed within the available time that is one sampling interval. Thus, these filters cannot efficiently be online adapted during frequency deviations. If we want to avoid the burden of these calculations, than a proper tabulation of the weights can be applied.

The proposed algorithm for estimation is based on the use of the values that were obtained as a result of the sampling of the continual input signal. This kind of processing must be done as many times as it is needed to enable the determination of the unknown signal parameters. The obtained system of the linear equations can be simply solved by using the derived analytical and summarized expressions. For this reason, the proposed method offers a significant improvement in computational efficiency over the standard reconstruction algorithms, at a lower numerical error. The method is designed for very accurate RMS measurements of periodic signals, and can be applied in precise measurements of other important quantities such as power and energy.

Unlike the IEEE standard that was analysed in [6], the algorithm proposed in this paper is significantly more stable and free of the propagation error. Namely, when using the procedure prescribed by the standard, the amplitude errors of the fundamental will propagate through the method since the amplitudes are used to reconstruct the detected sine wave and obtain the results before they are used to determine the next harmonic parameters. Overall, the frequency and amplitude errors from the first calculation are propagated to the higher harmonics and the calculation of the $n^{\text {th }}$ harmonic will invariably be contaminated by the errors of the phases and amplitudes from previous steps.

\section{SUGGESTED METHOD OF PROCESSING}

Let us assume that the input signal of the fundamental frequency $f$ is band-limited to the first $M$ harmonic component. This form of continuous signal with a complex harmonic content can be represented as a sum of the Fourier components as follows:

$$
s(t)=\sum_{k=1}^{M} a_{k} \sin \left(k 2 \pi f t+\psi_{k}\right)
$$

By sampling the signal (1), and by forming a system of equations of the same form, in order to determine the $2 M$ unknowns (amplitudes and phases of the $M$ harmonic), we obtain:

$$
s\left(t_{l}\right)=\sum_{k=1}^{M} a_{k} \sin \left(k 2 \pi f t_{l}+\psi_{k}\right)
$$

where $l=1,2, \ldots, 2 M$. The $t_{l}$ is time moment in which the sampling of the input analogue signal is done. The $s\left(t_{l}\right)$ value represents the value of the processed signal at the moment when the sampling is performed. The obtained relation can be represented in the short form as:

$$
\sum_{k=1}^{M} a_{k}\left(\sin \alpha_{k, l} \cos \psi_{k}+\cos \alpha_{k, l} \sin \psi_{k}\right)=s\left(t_{l}\right)
$$

where:

$$
\alpha_{k, l}=2 k \pi f t_{l}=k \alpha_{l} ; \quad(k=1,2, \ldots, M) ;(l=1,2, \ldots, 2 M)
$$

The $\alpha_{k, l}$ are the variables determined by the moment at which the sampling is done, as well as by the frequency of the corresponding harmonic of the input periodic signal.

If the input signal being processed contains a DC component, then this component can be simply separated (determined) by using an adequate filter, after which it is measured, before the signal itself is subjected to sampling. In this way, the reconstruction system suggested in this paper gives possibility to process the most general form of a periodic input signal.

\section{A. Frequency estimation}

The process of estimating the frequency of multi-sine wave signals, from a finite number of noisy discrete-time measurements, is an important task from both the theoretical and practical point of view. The problem has been the focus of research for quite some time and still is an active research area to date [7-11], since it is used in a wide range of applications in many fields such as control theory, relaying protection, intelligent instrumentation of power systems, signal processing, digital communications, distribution automation, biomedical engineering, radar applications, radio frequency, instrumentation and measurement, to name just a few. A list of several algorithms is reported: adaptive notch filter, time frequency representation based method, phase locked loop based method, eigensubspace tracking estimation, extended Kalman filter frequency estimation, and internal model based method. The requirements on the frequency estimator, and thus the choice of the solution, vary with the application, but typical issues are: accuracy, processing speed or complexity, and ability to handle multiple signals. This paper presents a method for estimating the frequencies of a multi (single)-sinusoidal signal, based on the detected zero-crossing (ZC) of the processed signal:

$$
s_{l} \cdot s_{l-1}<0
$$

The ZC method is widely used for its simplicity. However, its accuracy is influenced by ZC detection, quantisation error, harmonics, noise, etc... In addition, its tracking of frequency change is slow because a $\mathrm{ZC}$ can only be detected after at least half a cycle and it takes more cycles for stable results. As it has been defined by equation (5), the number of zero-crossings of the processed signal is evaluated by a 
sign-test of consecutive samples. This type of approach is radically different from the methods described in $[12,13]$, that are based on the use of either PLL or filtering of the harmonics and after-sampling, or sampling of the whole signal filtering of the harmonics and post-processing etc., which are more complicated than the threshold crossing technique proposed in this paper.

In order to increase the anti-noise capability, a $2 n$-length window of data can be used. A ZC is determined to be included in the window only when $n$ consecutive samples have positive signs and $n$ consecutive samples have negative signs. By taking $n=3 \ldots 5$, the erroneous $\mathrm{ZC}$ brought by random noise could be effectively reduced. After the $\mathrm{ZC}$ is detected, the ZC position on time axis can be located by linear interpolation with two consecutive samples that have different signs. Assuming the two samples are $s_{k}>0$ and $s_{k-}$ ${ }_{1}<0$, the location of a $\mathrm{ZC}$ on the time axis is computed by:

$$
x=k-\frac{s_{K}}{s_{K}-s_{K-1}}
$$

After the locations of two consecutive ZCs at $x_{1}$ and $x_{2}$ are known, the frequency is computed by:

$$
f=\frac{1}{2 T_{s}\left(x_{1}-x_{2}\right)}
$$

where $T_{S}=1 / f_{S}$ is the sampling interval. For online frequency estimation, a sliding window of samples is used so that the above $x_{1}$ and $x_{2}$ are referring to the start point of the data window. A security condition is also added to exclude erroneous ZC. That is, if the interval between two ZCs is significantly different from the previous interval, the newly detected $\mathrm{ZC}$ will be deemed invalid and the previous frequency result will be used instead. With this simple logic, the derived frequency accuracy is already sufficiently good. The idea was to develop a simpler algorithm of a low numerical complexity, capable of offering enough precision in determining the frequency of the processed signals. This was confirmed by the results of the checks that were performed on the suggested algorithm.

In a power system application, it is inevitable that signal may be contaminated by inter-harmonics and random noise, which would have adverse impact on any frequency estimation algorithms. Therefore, it is necessary to add prefilters before the main algorithm is executed.

\section{B. Proposed algorithm for signal parameter estimation}

The system determinant for the system of $2 M$ unknown parameters (equation (3)), can be represented as:

$$
\begin{aligned}
& \mathbf{X}_{2 M}=\left|\begin{array}{cccccccc}
\sin \alpha_{1,1} & \sin \alpha_{2,1} & \ldots & \sin \alpha_{M, 1} & \cos \alpha_{1,1} & \cos \alpha_{2,1} & \ldots & \cos \alpha_{M, 1} \\
\sin \alpha_{1,2} & \sin \alpha_{2,2} & \ldots & \sin \alpha_{M, 2} & \cos \alpha_{1,2} & \cos \alpha_{2,2} & \ldots & \cos \alpha_{M, 2} \\
\cdot & . & . & . & . & . & . & . \\
\sin \alpha_{1,2 M} & \cos \alpha_{2,2 M} & \ldots & \sin \alpha_{M, 2 M} & \cos \alpha_{1,2 M} & \cos \alpha_{2,2 M} & \ldots & \cos \alpha_{M, 2 M}
\end{array}\right| \\
& =\left|\begin{array}{cccccccc}
\sin \alpha_{1} & \sin 2 \alpha_{1} & \ldots & \sin M \alpha_{1} & \cos \alpha_{1} & \cos 2 \alpha_{1} & \ldots & \cos M \alpha_{1} \\
\sin \alpha_{2} & \sin 2 \alpha_{2} & \ldots & \sin M \alpha_{2} & \cos \alpha_{2} & \cos 2 \alpha_{2} & \ldots & \cos M \alpha_{2} \\
\cdot & . & . & . & . & . & . & . \\
\sin \alpha_{2 M} & \sin 2 \alpha_{2 M} & \ldots & \sin M \alpha_{2 M} & \cos \alpha_{2 M} & \cos 2 \alpha_{2 M} & \ldots & \cos M \alpha_{2 M}
\end{array}\right|
\end{aligned}
$$

A similar form of the obtained determinant (8) was the object of study in [14]. Unlike [14], this paper introduces a direct sampling of the processed signal, rather than its differentiating and sampling. This approach simplifies the methodology and reduces the possible mistake in the calculation of the unknown parameters. Apart from this, this kind of approach reduces the number of parameters dependent on the frequency of the processed signal [14] (the parameter $A_{k}$ does not occur), while the value of parameter $\alpha$ (relation (4)) and the very determinants are calculated in a slightly modified manner. In its form, determinant (8) resembles the well-known Van der Monde determinant. Owing to relations derived in this way [14], it is not necessary to use the standard procedure for solving the system of equations. This standard procedure, in the case of an extremely complex spectral content of a signal, would require a powerful processor and enough time for processing.

The given determinant can be solved as it was done in [14] (by using Euler's formulas):

$$
\begin{aligned}
& \mathbf{X}_{2 M}=(-1)^{\frac{M(M+1)}{2}} 2^{2 M(M-1)} \prod_{j=k+1}^{2 M} \prod_{k=1}^{2 M-1} \sin \frac{\alpha_{j}-\alpha_{k}}{2} \\
& \cdot \sum \cos \left\{\frac{\alpha_{1}+\alpha_{2}+\ldots+\alpha_{2 M}}{2}-\left(\alpha_{1}+\alpha_{2}+\ldots+\alpha_{2 M}\right)_{M}\right\}
\end{aligned}
$$

(the summing is done by all of the M's of the set $\left.\left\{\alpha_{1}, \alpha_{2}, \ldots, \alpha_{2 M}\right\}\right)$.

The co-determinants required for reaching the solution of the given system of equations (3) are:

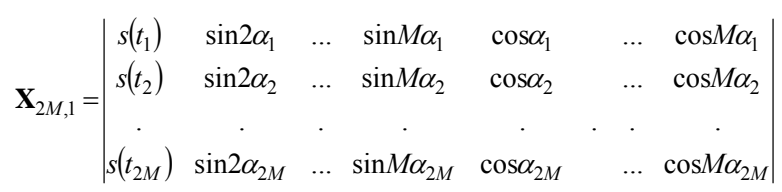

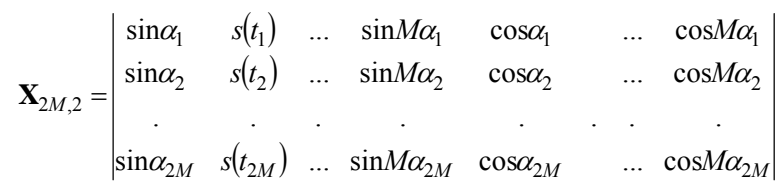

and so on. The co-determinants given above based on the following development can be written as:

$$
\mathbf{X}_{2 M, 1}=s\left(t_{1}\right) \mathbf{X}_{1}^{1}+s\left(t_{2}\right) \mathbf{X}_{2}^{1}+\ldots+s\left(t_{2 M}\right) \mathbf{X}_{2 M}^{1}
$$

$\mathbf{X}_{1}^{1}, \mathbf{X}_{2}^{1}, \ldots, \mathbf{X}_{2 M}^{1}$ are the co-determinants, obtained from co-determinant $\mathbf{X}_{2 M, 1}$ after the corresponding row as well as the first column had been eliminated. The second codeterminant (or co-factor) is derived from the expansion of $\mathbf{X}_{2 M}$ along such a column. For this purpose, we must determine $\mathbf{X}_{p}^{q}$ as co-factors of $\mathbf{X}_{2 M}$ [14]. Therefore: 


$$
\mathbf{X}_{p}^{q}=(-1)^{p+q} \mathbf{F}_{p}^{q}
$$

where $\mathbf{F}_{p}^{q}$ is obtained from $\mathrm{X}_{2 M}$ overturning $p$ row and $q$ column. We know that [14]:

$$
\Delta_{2 M+1, M+1}=\Delta_{2 M+1, M+1}^{(2 M+1)}
$$

where $\Delta$ is the Van der Monde determinant defined in [14].

$$
\mathbf{X}_{2 M=} \frac{(-1)^{\frac{M(M+1)}{2}}}{2^{M}} e^{-M \frac{\pi}{2} i} e^{-M\left(\alpha_{1}+\ldots+\alpha_{2 M}\right) i} \Delta_{2 M+1, M+1}\left(x_{t}=e^{\alpha_{t} i}\right)
$$

Hence, it follows that $\mathbf{X}_{p}^{q}$ is co-factor of matrix, whose determinant is $\Delta_{2 M+1, M+1}$.

$$
\Delta_{2 M+1, M+1}=\left(\prod_{j=k+1}^{2 M} \prod_{k=1}^{2 M-1}\left(x_{j}-x_{k}\right)\right)\left(x_{1} x_{2} \ldots x_{2 M}\right) \sum \frac{1}{\left(x_{1} x_{2} \ldots x_{2 M}\right)_{M}}
$$

(the sum of all inverted $M$ products of different indices).

After the intensive mathematical calculation [14] we obtain:

$$
\begin{aligned}
& \mathbf{F}_{p}^{q}=\frac{(-1)^{\frac{M(M+3)}{2}}}{2^{M}} e^{-M \frac{\pi}{2} i} e^{-M\left(\alpha_{1}+\ldots+\alpha_{p-1}+\alpha_{p+1}+\ldots+\alpha_{2 M}\right) i} . \\
& \cdot\left(\Delta_{2 M+1, M+1}^{(p, M+q)}-\Delta_{2 M+1, M+1}^{(p, M-q+1)}\right)_{x_{j}=e^{\alpha_{j i} i}}
\end{aligned}
$$

for $1 \leq p \leq 2 M \wedge 1 \leq q \leq M$.

$$
\begin{aligned}
& \mathbf{F}_{p}^{q}=\frac{(-1)^{\frac{M(M+3)}{2}}}{2^{M}} e^{-(M-1) \frac{\pi}{2} i} e^{-M\left(\alpha_{1}+\ldots+\alpha_{p-1}+\alpha_{p+1}+\ldots+\alpha_{2 M}\right) i} . \\
& \cdot\left(\Delta_{2 M+1, M+1}^{(p, M+q)}-\Delta_{2 M+1, M+1}^{(p, M-q+1)}\right)_{x_{j}=e^{\alpha_{j} i}}
\end{aligned}
$$

for $1 \leq p \leq 2 M ; M+1 \leq q \leq 2 M$, and $\mathbf{F}_{p}^{M+q}$ for $1 \leq q \leq M$.

The results obtained from solving of the observed determinants were compared with the solution obtained from GEPP algorithm (Gaussian elimination with partial pivoting), offered in the Matlab program package itself (all of the calculations are done in IEEE standard double floating point arithmetic with unit round off $u \approx 1.1 \times 10^{-16}$ ). This represents a practical verification of the proposed algorithm for a case of ideal sampling (without an error in taking the value of the sample and determining the frequency of the processed signal). The difference in the obtained values and results obtained with GEPP algorithm were equal to $1 \times 10^{-14}$. The derived relations produce solutions that are practically identical to the procedure that is most commonly used in solving systems of linear equations.

Based on relation derived in this way, the unknown parameters of the signal (amplitude, phase) can be determined through a simple division of the expression that represents a solution of the adequate co-determinants with the expression that represents an analytical solution to the system determinant:

$$
\begin{aligned}
& \psi_{k}=\operatorname{arctg} \frac{\mathbf{X}_{M+k}}{\mathbf{X}_{k}} \\
& a_{k}=\frac{1}{\mathbf{X}_{2 M}} \sqrt{\mathbf{X}_{k}^{2}+\mathbf{X}_{M+k}^{2}}
\end{aligned}
$$

It is a fact that the obtained system of equations (3) can be described, after the processing, by a special form of the determinant (which is summarized as the Van der Monde determinant). This fact enables factoring and application of transformations that can be applied only on determinants. Any other procedure would lead to a much more complex calculation and to relations that are mathematically much more demanding. If the sampled real signal has interharmonic components, the proposed mathematical transformations are not correct anymore.

\section{PROPOSED ReCONSTRUCTION ALGORITHM}

When using the proposed algorithm, the first step to be taken is to ensure that the order of the highest $M$ harmonic component in the processed signal spectrum is adopted in advance, accepting that an $M$ determined in this way is bigger than the expected (real) value. The $M$ determined in this way can be corrected after each passage through the proposed procedure and adapted according to the actual harmonic content of the input processing signal, which enables additional estimation optimization. After this step, it is possible to perform the procedure of determining the frequency of the basic harmonic, using the previously described methodology and based on the detected zerocrossings.

In order to recalculate unknown parameters (amplitude and phase) of the processed periodic signals, it is necessary to have the results of the sampling of the input analogue signals $s\left(t_{t}\right)$, (equation (2)). The sampling of the input signal must be done in $2 M$ points, so as to be able to recalculate all of the unknown values. The samples of the input signal are obtained by the means of sampling at precisely defined time moments, which are referred to in relation to the detected moment of zero crossing. The values of the derived expressions depend on the measured frequency $f$, because the values of the determinant elements are calculated based on coefficient $\alpha_{l}$, according to equation (4). Apart from this, other parameters of the derived system of equations will not be dependent on the frequency of the carrier. 
With every passage of the described algorithm, the moment of sampling is referred to the detected zero-crossing of the processed signal, and its basic frequency is also calculated at the same time. In this way, the determination of the unknown parameters of the processed periodic signal is less dependent on the possible frequency mismatch. The algorithm proposed here is much less sensitive to the variation in the frequency of the carrier signal. The moments $t_{l}$ in which the sampling of the input signal is done can be completely random (asynchronous) and independent of the frequency of the processed signal, due to the way in which they are defined in equation (4). The interval between two consecutive samples is actually dependent primarily on the speed of the $\mathrm{S} / \mathrm{H}$ (sample and hold) circuit and the $\mathrm{AD}$ (analogue to digital) conversion circuit, with which a numeric equivalent to the sample of the input signal is formed. However, due to the practical realization and the way in which real sigma-delta ADC function, the $t_{l}$ moments can be defined as $t_{l}=l t_{\text {sample }}$, where $t_{\text {sample }}=1 / f_{S}\left(f_{S}\right.$ is the sampling frequency).

Due to the presence of the error in determining the samples $s\left(t_{l}\right)$, and variables $\alpha_{l}$, which is caused by their dependence on the carrier frequency $f$ of the processed signal, in the practical applications of the proposed algorithm we need to have the best estimate of the given values, according to the criterion assumed. This can be done by the means of recalculation of the values $s\left(t_{l}\right)$ and $\alpha_{l}$, through $N$ passages, ( $N$ is arbitrary). In this process we form series $s\left(t_{l}\right)_{i}$ and $\alpha_{n i}(i=1, \ldots, N)$, as given in the proposed algorithm. The random errors $\Delta_{n}$ of measurements are unbiased $E\left(\Delta_{i}\right)=0$, have the same variance $\operatorname{var}\left(\Delta_{i}\right)=\sigma^{2}$, and are not mutually correlated. Under these assumptions, we can use the weighted average procedure for decreasing random errors in determination of observed values. The weighted average is used for measurements that are not correlated and have a varying degree of accuracy. The averages $\hat{s}\left(t_{l}\right), \hat{\alpha}_{l}$ of the values $s\left(t_{l}\right)$, and $\alpha_{l}$ are calculated for all $l=1,2, \ldots, 2 M$ as:

$$
\hat{s}\left(t_{l}\right)=\frac{\sum_{i=1}^{n_{s}} w_{s i} s\left(t_{l}\right)_{i}}{\sum_{i=1}^{n_{s}} w_{s i}} ; \hat{\alpha}_{l}=\frac{\sum_{n=1}^{n_{\alpha}} w_{\alpha i} \alpha_{l i}}{\sum_{i=1}^{n_{\alpha}} w_{\alpha i}} ; \sum_{i=1}^{n_{s}} w_{s i}=\sum_{i=1}^{n_{\alpha}} w_{\alpha i}=N
$$

where $w_{s i}, w_{\alpha i}$, are non-negative weights of series $s\left(t_{l}\right)_{i}, \alpha_{l i}$. The $n_{s}, n_{\alpha}$, defines the numbers of different values in the above series through $N$ passages. The value of $N$ will depend on the required speed of processing - the higher the $N$, the more precise the estimation of the value is. In this particular case, the estimation procedure does not require the matrix inversion and is considerably less demanding from the processor aspect. The proposed solution can be modified, in order to reduce the error in determining the samples of the input signal. In [15] it was shown that implementation of sampling and reconstruction with internal antialiasing filtering radically improves performances of digital receivers, enabling reconstruction with a much lower error.
After using the procedure described above to perform the estimation of the value of samples $s\left(t_{l}\right)$ and variables $\alpha_{l}$, it is necessary to perform the recalculation of the unknown amplitudes and phases, for all of the harmonic components of the processed band-limited periodic signal. According to the Fourier coefficients determined in this way, it is possible to perform the calculation of the effective value of the signal, the active power and energy. When this is done, it is possible to start the sampling of the processed signal again.

\section{Computing time}

The suggested algorithm can be applied in operation with sigma-delta ADC, thus enabling high resolution and speed in processing of input signals. The time needed to perform the necessary number of samplings of the input signal that is the object of reconstruction is defined as $2 M t_{\text {sample }}$, which represents the value approximate to the time needed for reconstruction (in simulation). In practical applications of the proposed algorithm, the determined time for the reconstruction of the processing signal ought to be increased by the time necessary to estimate the variables $s\left(t_{l}\right)$, and $\alpha_{l}$ (this time is directly dependent on the value $N$ ), and the time interval $\Delta t$, which is necessary to perform all the other recalculations according to the proposed algorithm. Considering all of the facts presented above, the reconstruction time can be defined as $N \cdot 2 M \cdot t_{\text {sample }}+\Delta t \approx N / f+\Delta t, \quad$ because of the necessary synchronization with the zero crossing of the input signal. The speed of the proposed algorithm makes it as fast as the algorithms analyzed in [16, 17].

In order to demonstrate the efficiency of the new procedure there is a comparison of the computing time of the proposed algorithm to GEPP algorithm in solving the system of equations (3), Table1 (using Matlab program package, version R2010b). The circumstance of verifying real-time characteristic is in computer with Intel Pentium 2.0G Dual CPU, 2Gb RAM, and Windows XP 2002 operation system. The results given in Table1 practically present the estimated value of the time interval $\Delta t$. The proposed procedure shortens the time needed for calculation by 2 to 3 times, depending on the number of the harmonic components of the processed signal. With a more powerful hardware platform and a different program environment, the time for the realization of the proposed algorithm will be many times shorter.

The paper [18] gives a measurement of the required processor time, in the realization of the matrix method in the reconstruction of signals, in the form in which it is implemented in many program packages. The method suggested by this paper does not require any special memorization of the transformation matrix, nor does it require recalculation of the inversion matrix. In this way, it is much more efficient in implementation and it is not limited only to sparse matrices. In addition to this, the proposed solution becomes easier for hardware realization, while the proposed algorithm can be practically implemented on any platform. 
Table1. Comparison of the computing time.

\begin{tabular}{|c|c|c|}
\hline $\begin{array}{c}\text { Number of } \\
\text { harmonic } \\
\text { components, } M\end{array}$ & $\begin{array}{c}\text { Proposed } \\
\text { algorithm }\end{array}$ & GEPP algorithm \\
\hline 5 & $0.00084 \mathrm{~s}$ & $0.00171 \mathrm{~s}$ \\
\hline 7 & $0.00091 \mathrm{~s}$ & $0.00215 \mathrm{~s}$ \\
\hline 8 & $0.00098 \mathrm{~s}$ & $0.00251 \mathrm{~s}$ \\
\hline 9 & $0.00104 \mathrm{~s}$ & $0.00297 \mathrm{~s}$ \\
\hline 11 & $0.00119 \mathrm{~s}$ & $0.00399 \mathrm{~s}$ \\
\hline 15 & $0.00173 \mathrm{~s}$ & $0.00564 \mathrm{~s}$ \\
\hline
\end{tabular}

\section{Simulation Results}

The algorithm proposed in this paper is tested by means of the input data obtained through computer simulation. First, an input-frequency modulated sinusoidal test signal (step frequency change from 50 to $49.8 \mathrm{~Hz}$ at $t=0 \mathrm{~s}$ ) was processed. A white noise with SNR $=60 \mathrm{~dB}$ was added to the sinusoidal signal. The test has been developed with the distorted source voltage (10\% third, $5 \%$ fifth, 3\% seventh and $2 \%$ eleventh harmonics). The phase angles of each harmonic were randomly chosen. The results obtained confirm a good dynamic response of the algorithm for the frequency step change and accuracy. The proposed algorithm is capable of adaptively tracking time variations of the characteristics of the power signal over time. It is observed that apart from a brief transient at the time of the step change, the algorithm effectively follows the variations in frequency. As shown in Fig.1, we have obtained a technique that provides accurate frequency estimation with error in the range of $0.001 \mathrm{~Hz}$.

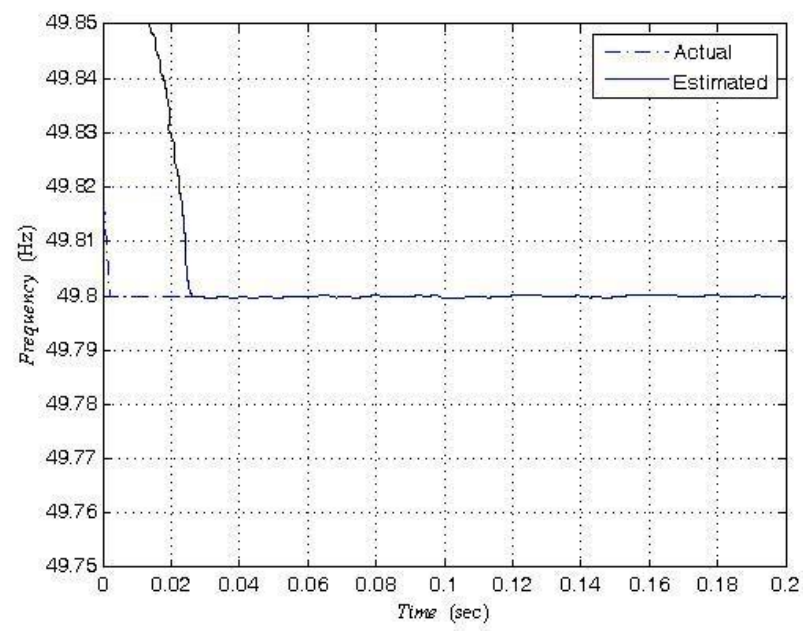

Fig.1. Estimation for $f=50 \mathrm{~Hz}$ for $t<0 \mathrm{~s}$ and $f=49.8 \mathrm{~Hz}$ for $t>0 \mathrm{~s}$ with $\mathrm{SNR}=60 \mathrm{~dB}$ and with harmonics presence.

The ability of the frequency estimation over a wide range of frequency changes is investigated using sinusoidal test signals with the following time dependence $f(t)=50+0.5 \sin (10 \pi t)$ as shown in Fig.2. The good dynamic responses can be noticed. Considering the case simulates extreme conditions in a power system, the error can be accepted by most applications. If additional filters such as high-order IIR (Infinite Impulse Response) filters or moving average filters are applied, the influence of noise can be further mitigated at the price of filter delay.

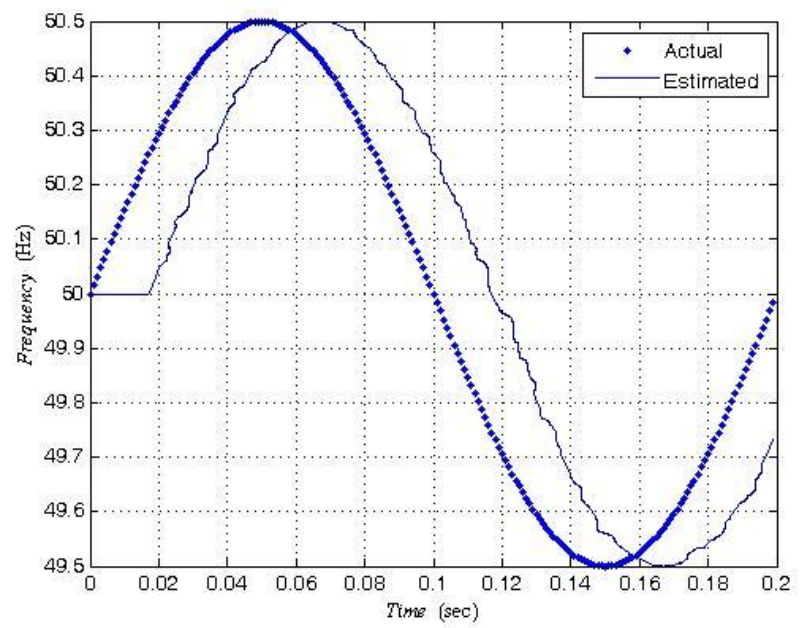

Fig.2. Estimation for $f(t)=50+0.5 \sin (10 \pi t)$ with $\mathrm{SNR}=60$ $\mathrm{dB}$ with harmonics presence.

The effect of noise presence in the signal was studied by estimating the frequency and magnitude of signals that contain noise. A sinusoidal $50 \mathrm{~Hz}$ input test signal with superimposed additive white centred Gaussian noise was used as input for the test. The random noise was selected to obtain a prescribed value of the SNR, which is defined as $\mathrm{SNR}=20 \log (A / \sqrt{2} \sigma)$, where $A$ is the magnitude of the signal fundamental harmonics, and $\sigma$ is the noise standard deviation. Fig. 3 shows the maximum errors observed in frequency and harmonic magnitude estimates when input signals of 30,50, and $70 \mathrm{~Hz}$ having SNRs of 40,50, 60, and $70 \mathrm{~dB}$ were used. It should be noted that, in practice, the SNR of the voltage signal obtained from a power system ranges between 50 and $70 \mathrm{~dB}$. At this level of noise, very little error is expected with the proposed technique, as depicted in Fig.3.

In order to investigate the statistical properties of the proposed estimator, noisy samples generated by computer simulation are used. Noisy samples are obtained by adding white noise samples to the samples of processing signal. For the estimation of deterministic parameters, a commonly used lower bound for the mean square error (MSE) is the Cramer-Rao lower bound (CRLB), given by inverse of the Fisher information [19-21]. Fig.4 and 5 depict the MSE of the amplitudes and frequency after $10^{5}$ simulations, respectively. Results clearly show that the proposed estimation schemes asymptotically reach the CRLB as in $[21,22]$. 

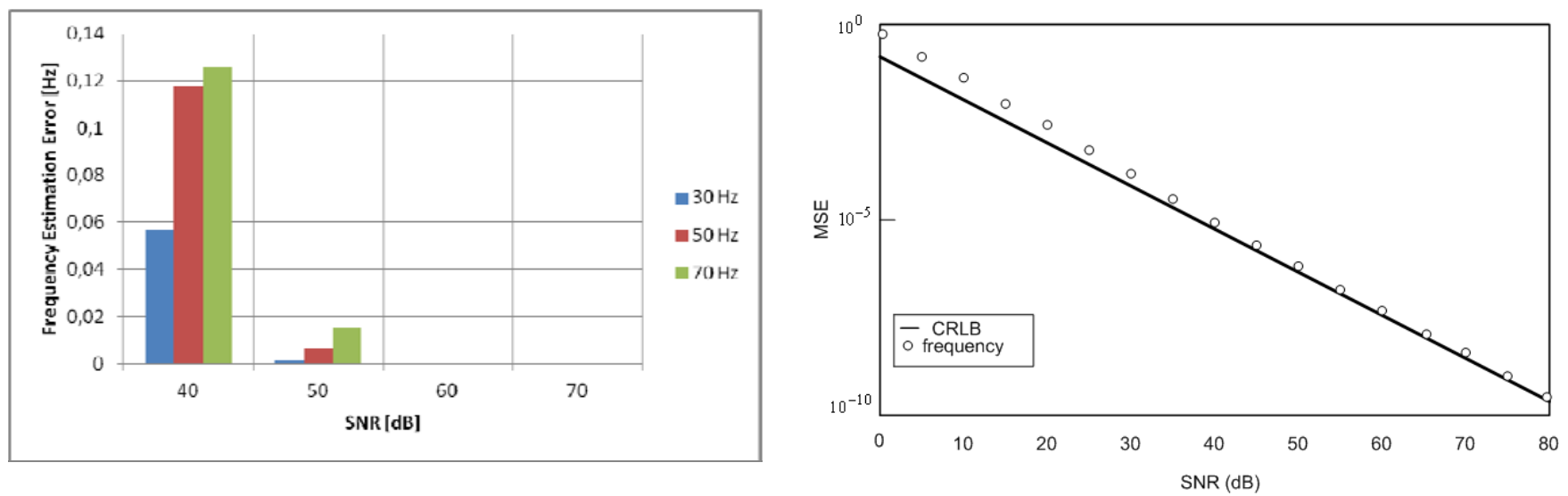

Fig.4. MSE of the frequency as a function of SNR.
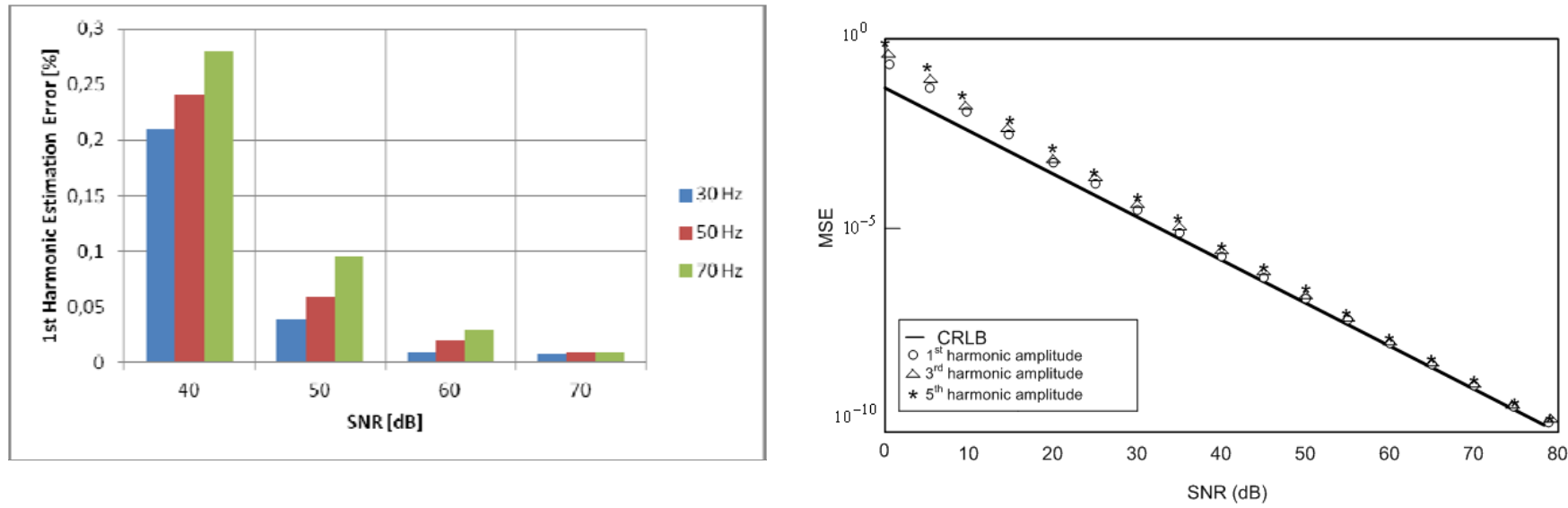

Fig.5. MSE of the three harmonic amplitudes as a function of SNR.
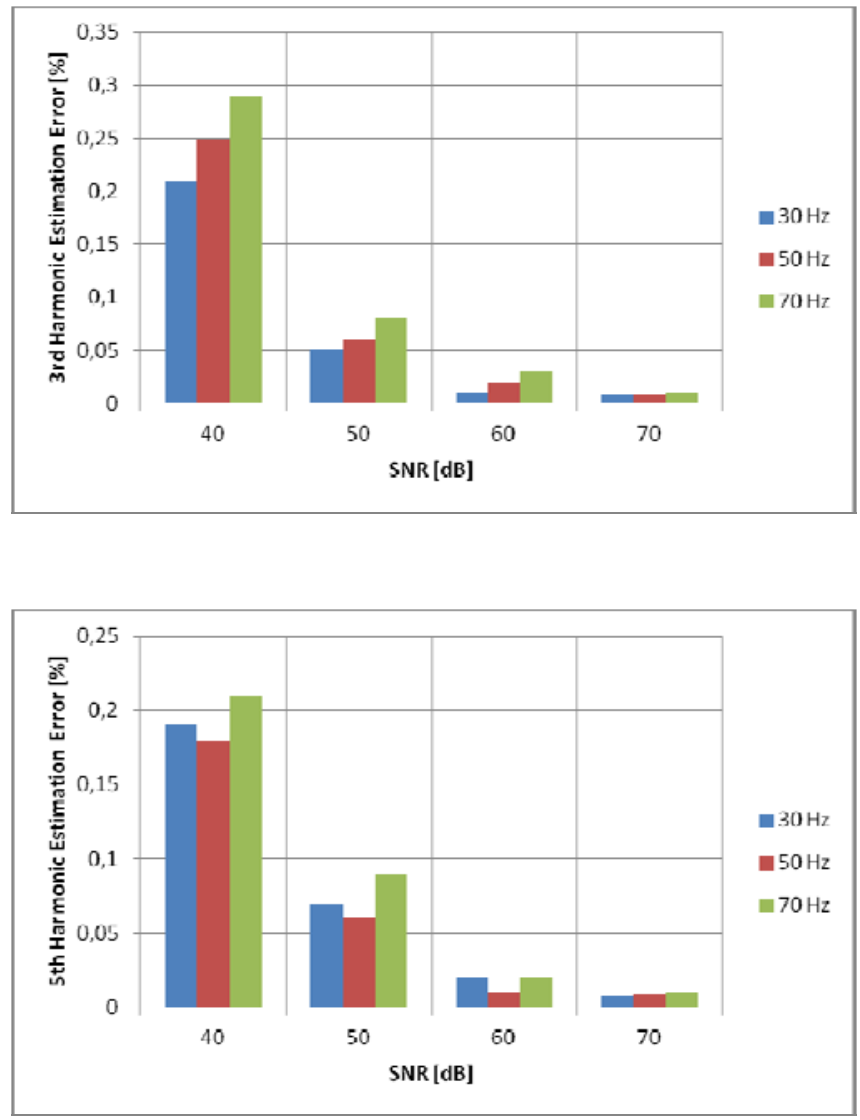

Fig.3. Maximum estimation errors for noisy input signals.

Table2. Comparison of simulation results by the proposed reconstruction algorithm, FFT and continuous wavelet transformation (CWT) [18]

\begin{tabular}{|c|c|c|c|c|}
\hline \multirow{2}{*}{$\begin{array}{l}\text { Harmonic } \\
\text { number }\end{array}$} & \multirow{2}{*}{$\begin{array}{l}\text { Amplitude } \\
\text { [VPP] }\end{array}$} & \multirow{2}{*}{$\begin{array}{l}\text { Phase } \\
\text { [rad] }\end{array}$} & \multicolumn{2}{|c|}{$\begin{array}{l}\text { Proposed reconstruction } \\
\text { algorithm }\end{array}$} \\
\hline & & & $\begin{array}{c}\text { Amp.error } \\
{[\%]}\end{array}$ & $\begin{array}{c}\text { Phase error } \\
{[\%]}\end{array}$ \\
\hline 1 & 1 & $\pi$ & 0.0018 & 0.0019 \\
\hline 2 & 0.81 & $\pi / 3$ & 0.0024 & 0.0022 \\
\hline 3 & 0.62 & 0 & 0.0022 & 0.0021 \\
\hline 4 & 0.58 & $\pi / 6$ & 0.0015 & 0.0016 \\
\hline 5 & 0.41 & $\pi / 4$ & 0.0015 & 0.0021 \\
\hline 6 & 0.33 & $\pi / 12$ & 0.0021 & 0.0019 \\
\hline 7 & 0.16 & 0 & 0.0020 & 0.0022 \\
\hline \multirow[t]{2}{*}{$\begin{array}{l}\text { Harmonic } \\
\text { number }\end{array}$} & \multicolumn{2}{|c|}{$\begin{array}{c}\text { FFT (sampling rate }=25 \\
\mathrm{kHz} ; \\
\text { data length }=25000 ; \\
\text { time period }=1 \mathrm{~s} \text { ) } \\
\end{array}$} & \multicolumn{2}{|c|}{ CWT } \\
\hline & $\begin{array}{c}\text { Amp.error } \\
{[\%]}\end{array}$ & $\begin{array}{l}\text { Phase } \\
\text { error [\%] }\end{array}$ & $\begin{array}{c}\text { Amp.error } \\
{[\%]}\end{array}$ & $\begin{array}{l}\text { Phase } \\
\text { error [\%] }\end{array}$ \\
\hline 1 & 0.296 & 0.322 & 0.023 & 0.034 \\
\hline 2 & 0.035 & 0.038 & 0.032 & 0.028 \\
\hline 3 & 0.875 & 0.843 & 0.049 & 0.026 \\
\hline 4 & 0 & 0 & 0.144 & 0.012 \\
\hline 5 & 0 & 0 & 0.013 & 0.154 \\
\hline 6 & 0 & 0 & 0.012 & 0.017 \\
\hline 7 & 0 & 0 & 0.223 & 0.186 \\
\hline
\end{tabular}


Additional testing of the proposed algorithm was carried out by simulation in the program package Matlab and SIMULINK. Standard sigma-delta ADC with the effective resolution of $24 \mathrm{bit}$, and sampling rate $f_{S}=1 \mathrm{kHz}$ was used as the ADC. During the simulation, the parameters of the input signal corresponded to the values given in Table2. The execution time of the proposed algorithm on hardware platform described earlier was $0.0167 \mathrm{~s}$. In the course of the simulation conducted in this way, the output PSD (Power Spectral Density) of the ideal, thermal noise affected and clock jitter affected was in the range of -100 to $-170 \mathrm{~dB}$ for the signal-to-noise distortion ratio (SNDR) ranging between $55 \mathrm{~dB}$ and $76 \mathrm{~dB}$.

A signal containing the first 7 harmonics was used, with the fundamental frequency $f=50 \mathrm{~Hz}$. The superposed noise and jitter will, in simulation performed in this way, cause a relative error in detection on fundamental frequency of $0.001 \%$. It can be seen that the accuracy of the proposed algorithm is within the limits that are attained in processing a signal of this form, in [17, 21, 23-25], and better then the one presented in [26]. In the time domain, the relative error between the signal and its reconstruction was $0.0025 \%$. The errors in the amplitude and phase detection are mainly due to the error in measuring of the input signal samples and the error in determining the value of the derived equations.

\section{Experimental results}

In order to verify the results obtained by simulation, experiments were conducted, using a laboratory prototype of "star" connected induction machine. Control structure consists of six thyristors, while each pair of back-to-back connected thyristors is connected between the phase source voltage and the appropriate phase of the induction machine, in the "star" connection. The parameters of the induction machine are: wound rotor type, two pole pairs, with: $R_{S}=5.2 \Omega, R_{r}=14.63 \Omega, L_{S}=L_{r}=0.055 H, L_{m}=1.3 H$. The firing angle $\alpha$ is defined as the angle between zero crossings of the phase voltage to the conduction beginning of the appropriate thyristor.

The experimental setup consists of voltage and current transducers, a connector block, data acquisition card and processing computer (PC). An acquisition card NI ELVIS/PCI-6251 Bundle (National Instruments) is used to input the current and voltage signals at each phase in the PC, where they are subjected to the proposed algorithm for processing. LEM HTR current and LEM CV3 voltage transducers were used as measurement hardware. The DAQ card sampling rate was $250 \mathrm{kS} / \mathrm{s}$.

For $n=1500 \mathrm{rpm}$ (the synchronous speed of the machine), "star" connection, the parameters of current and voltage harmonics in phase ' $a$ ' were recorded using the existing software (NI LabVIEW), Table3. The signals with this harmonic content were also processed using the proposed algorithm for the estimation of signal parameters. The difference in the amplitude and phase detection between the data acquisition card and proposed reconstruction algorithm are also given in Table3. The analysis shows that the proposed algorithm remains highly accurate in processing of periodic signals in real environment.
Table3. Comparison of experimental results by the proposed reconstruction algorithm and results obtained with acquisition card NI ELVIS/PCI-6251 Bundle

\begin{tabular}{|c|c|c|c|c|}
\hline \multirow[t]{2}{*}{ Parameters } & \multicolumn{2}{|c|}{$\begin{array}{l}\text { Acquisition card } \\
\quad\left(\alpha=110^{\circ}\right)\end{array}$} & \multicolumn{2}{|c|}{$\begin{array}{c}\text { Proposed reconstruction } \\
\text { algorithm } \\
\left(\alpha=110^{\circ}\right)\end{array}$} \\
\hline & $\begin{array}{l}\text { Measured } \\
\text { values }\end{array}$ & $\begin{array}{l}\text { Phase } \\
\text { (deg) }\end{array}$ & $\begin{array}{l}\text { Amplitude } \\
\text { differe. [\%] }\end{array}$ & $\begin{array}{c}\text { Phase } \\
\text { differe. [\%] }\end{array}$ \\
\hline $\begin{array}{c}\mathrm{I}_{\mathrm{ph} \text { a1 }}\left(1^{\mathrm{st}}\right. \\
\text { harmonic) }(\mathrm{A})\end{array}$ & 0.63 & 57.87 & 0.0021 & 0.0024 \\
\hline $\begin{array}{c}\mathrm{I}_{\mathrm{ph} \_\mathrm{a} 3}\left(3^{\text {rd }}\right. \\
\text { harmonic) (A) }\end{array}$ & 0 & 0 & 0.0019 & 0.0025 \\
\hline $\begin{array}{c}\mathrm{I}_{\mathrm{ph} \_\mathrm{a} 5}\left(5^{\text {th }}\right. \\
\text { harmonic) }(\mathrm{A})\end{array}$ & 0.13 & -21.77 & 0.0026 & 0.0022 \\
\hline $\begin{array}{c}\mathrm{I}_{\mathrm{ph} \_\mathrm{a} 7}\left(7^{\text {th }}\right. \\
\text { harmonic) }(\mathrm{A})\end{array}$ & 0.04 & -80.21 & 0.0018 & 0.0028 \\
\hline $\begin{array}{c}\mathrm{I}_{\mathrm{ph} \_ \text {a11 }}\left(11^{\text {th }}\right. \\
\text { harmonic })(\mathrm{A})\end{array}$ & 0.1 & 154.85 & 0.0018 & 0.0021 \\
\hline $\begin{array}{c}\mathrm{I}_{\mathrm{ph} \_ \text {a13 }}\left(13^{\text {th }}\right. \\
\text { harmonic) }(\mathrm{A})\end{array}$ & 0.114 & 25.47 & 0.0013 & 0.0023 \\
\hline $\begin{array}{c}\mathrm{I}_{\mathrm{ph} \_ \text {a17 }}\left(17^{\mathrm{th}}\right. \\
\text { harmonic) (A) }\end{array}$ & 0.02 & -12.63 & 0.0029 & 0.0017 \\
\hline $\begin{array}{c}\mathrm{I}_{\mathrm{ph} \_ \text {a19 }}\left(19^{\text {th }}\right. \\
\text { harmonic) }(\mathrm{A})\end{array}$ & 0.029 & -56.48 & 0.0023 & 0.0027 \\
\hline $\begin{array}{c}\mathrm{V}_{\text {out_al }}\left(1^{\text {th }}\right. \\
\text { harmonic) }(\mathrm{V})\end{array}$ & 271.94 & 0 & 0.0018 & 0 \\
\hline $\begin{array}{c}\mathrm{V}_{\text {out_a3 }}\left(3^{\text {rd }}\right. \\
\text { harmonic) }(\mathrm{V})\end{array}$ & 33.62 & 122.23 & 0.0028 & 0.0023 \\
\hline $\begin{array}{c}\mathrm{V}_{\text {out_a5 }}\left(5^{\text {th }}\right. \\
\text { harmonic) }(\mathrm{V})\end{array}$ & 23.12 & -42.37 & 0.0017 & 0.0026 \\
\hline $\begin{array}{c}\mathrm{V}_{\text {out_a7 }}\left(7^{\text {th }}\right. \\
\text { harmonic) }(\mathrm{V})\end{array}$ & 10.52 & 74.68 & 0.0017 & 0.0023 \\
\hline
\end{tabular}

\section{CONCLUSION}

The estimation procedure proposed in this paper is a new complexity-reduced algorithm for estimation of the frequency and signal parameters. The derived analytical expression opens a possibility to perform fast calculations with a low numeric error. The computing time is determined by the time that is necessary for the collection of the required number of samples of the processed signal and the estimation procedure itself. In any case, the time is much shorter than with any other known matrix method used. All the necessary hardware resources can be satisfied by a DSP of standard features and real sigma-delta ADC. Based on the identified parameters of the AC signals, we can establish all the relevant values in the electric utilities (energy, power, RMS values). The measurement uncertainty is a function of the error in synchronization with fundamental frequency of processing signal (because of the non-stationary nature of the jitter-related noise and white Gaussian noise), and the error that occurs in determining the values of the samples of the processed signal. Through computer simulations, it is shown that the mean square frequency and amplitude errors of the developed method can attain CRLB for sufficiently high signal-to-noise ratios. The simulation and experimental results show that the proposed algorithm can offer satisfactory precision in reconstruction of periodic signals in a real environment.

\section{ACKNOWLEDGMENT}

The author wishes to thank to the Ministry of Education and Science of the Republic of Serbia for its support of this work provided within the projects 42009 and OI-172057. 


\section{REFERENCES}

[1] Wang, F., Bollen, M. (2004). Frequency response characteristics and error estimation in RMS measurement. IEEE Trans. Power Delivery, 19 (4), 1569-1578.

[2] Wand, M., Sun, Y. (2004). A practical, precise method for frequency tracking and phasor estimation. IEEE Trans. Power Delivery, 19 (4), 1547-1552.

[3] Agrež, D. (2010). Estimation and tracking of the power quality disturbances in the frequency domain. Meas. Sci. Rev., 10 (6), 189-194.

[4] Terzija, V.V. (2003). Improved recursive Newton-type algorithms for frequency and spectra estimation in power systems. IEEE Trans. Instrum. Meas., 52 (5), 1654-1659.

[5] Sidhu, T.T. (1999). Accurate measurement of power system frequency using a digital signal processing technique. IEEE Trans. Instrum. Meas., 48 (1), 75-81.

[6] Arpaia, P., Cruz Serra, A., Daponte, P., Monteiro, C.L. (2001). A critical note to IEEE 1057-94 standard on hysteretic ADC dynamic testing. IEEE Trans. Instrum. Meas., 50 (4), 941-948.

[7] Wu, J., Long, J., Wang, J. (2005). High-accuracy, wide-range frequency estimation methods for power system signals under nonsinusoidal conditions. IEEE Trans. Power Delivery, 20 (1), 366-374.

[8] So, H.C., Chan, K.W., Chan, Y.T., Ho, K.C. (2005). Linear prediction approach for efficient frequency estimation of multiple real sinusoids: Algorithms and analyses. IEEE Trans. Signal Process., 53 (7), 2290-2305.

[9] Klein, J.D. (2006). Fast algorithms for single frequency estimation. IEEE Trans. Signal Process., 54 (5), 1762-1770.

[10] El-Shafey, M.H., Mansour, M.M. (2006). Application of a new frequency estimation technique to power systems. IEEE Trans. Power Delivery, 21 (3), 1045-1053.

[11] Trapero, J.R., Sira-Ramirez, H., Batlle, V.F. (2007). An algebraic frequency estimator for a biased and noisy sinusoidal signal. Signal Process., 87 (6), 1188-1201.

[12] Martens, O., Trampark, H., Liimets, A., Nobel, P., Veskimester, A., Jarvalt, A. (2008). DSP-based power quality monitoring device. In IEEE International Symposium on Intelligent Signal Processing (WISP 2007), 3-5 October 2007. IEEE, 1-5.

[13] Hindersah, H., Purwadi, A., Ali, F.Y., Heryana, N. (2011). Prototype development of single phase prepaid KWh meter. In International Conference on Electrical Engineering and Informatics, 17-19 July 2011. IEEE, 1-6.
[14] Petrovic, P. (2010). Fourier coefficient estimation based on the differential values of the processed signal. IETE Jornal of Research, 56 (1), 30-43.

[15] Poberezhskiy, Y.S., Poberezhskiy, G.Y. (2004). Sampling and signal reconstruction circuits performing internal antialiasing filtering and their influence on the design of digital receivers and transmitters. IEEE Trans. Circ. Syst.-I, 51 (1), 118-129.

[16] Cooklev, T. (2006). An efficient architecture for orthogonal wavlet transforms. IEEE Signal Process. Lett., 13 (2), 77-79.

[17] Schoukens, J., Rolain, Y., Simon, G., Pintelon, R. (2003). Fully automated spectral analysis of periodic signals. IEEE Trans. Instrum. Meas., 52 (4), 1021-1024.

[18] Reeves, S.J. (1999). An efficient implementation of the backward greedy algorithm for sparse signal reconstruction. IEEE Signal Process. Lett., 6 (10), 266-268.

[19] Kay, S.M. (1988). Modern Spectral Estimation: Theory and Applications. Englewood Cliffs, NJ: Prentice-Hall.

[20] Stoica, P., Li, H., Li, J. (2000). Amplitude estimation of sinusoidal signals: Survey, new results, and an application. IEEE Trans. Signal Process., 48 (2), 338-352.

[21] Belega, D., Dallet, D., Slepicka, D. (2010). Accurate amplitude estimation of harmonic components of incoherently sampled signals in the frequency domain. IEEE Trans. Instrum. Meas., 59 (5), 1158-1166.

[22] Pantazis, Y., Roces, O., Stylianou, Y. (2010). Iterative estimation of sinusoidal signal parameters. IEEE Signal Process. Lett., 17 (5), 461-464.

[23] Hidalgo, R.M., Fernandez, J.G., Rivera, R.R., Larrondo, H.A. (1996). A simple adjustable window algorithm to improve FFT measurements. IEEE Trans. Instrum. Meas., 51 (1), 31-36.

[24] Agrež, D. (2002). Weighted multi-point interpolated DFT to improve amplitude estimation of multifrequency signal. IEEE Trans. Instrum. Meas., 51 (2), 287-292.

[25] Agrež, D. (2005). Improving phase estimation with leakage minimization. IEEE Trans. Instrum. Meas., 54 (4), 1347-1353.

[26] Tse, N.C.F., Lai, L.L. (2007). Wavelet-based algorithm for signal analysis. EURASIP Journal on Advances in Signal Processing, 2007 (1), art. ID 38916.

Received January 24, 2012. Accepted September 14, 2012. 